Proceedings of the 2011 Villa de Leyva Summer School

\title{
Geometric, Algebraic and Topological Methods for Quantum Field Theory
}


This page intentionally left blank 
Proceedings of the 2011 Villa de Leyva Summer School

\author{
Geometric, Algebraic and \\ Topological Methods \\ for Quantum Field Theory \\ Villa de Leyva, Colombia $\quad 4-22$ July 2011
}

Editors

Alexander Cardona

Universidad de los Andes, Colombia

Carolina Neira-Jiménez Universität Regensburg, Germany

Hernán Ocampo

Universidad del Valle, Colombia

Sylvie Paycha

Universität Potsdam, Germany

Andrés F Reyes-Lega

Universidad de los Andes, Colombia 
Published by

World Scientific Publishing Co. Pte. Ltd.

5 Toh Tuck Link, Singapore 596224

USA office: 27 Warren Street, Suite 401-402, Hackensack, NJ 07601

UK office: 57 Shelton Street, Covent Garden, London WC2H 9HE

\section{Library of Congress Cataloging-in-Publication Data}

Villa de Leyva Summer School (7th : 2011 : Leiva, Boyacá, Colombia)

Geometric, algebraic and topological methods for quantum field theory : proceedings of the 2011 Villa de Leyva Summer School, Villa de Leyva, Colombia, 4-22 July 2011 / edited by Alexander Cardona (Universidad de los Andes, Colombia), Carolina Neira-Jiménez (Universität Regensburg, Germany), Hernán Ocampo (Universidad del Valle, Colombia), Sylvie Paycha (Universität Potsdam, Germany) \& Andrés F Reyes-Lega (Universidad de los Andes, Colombia). pages $\mathrm{cm}$ Includes bibliographical references and index.

ISBN 978-981-4460-04-0 (hardcover : alk. paper)

1. Geometric quantization--Congresses. 2. Quantum field theory--Mathematics--Congresses.

3. Topology--Congresses. I. Cardona, Alexander, editor of compilation. II. Title. QC174.17.G46G455 2011

530.14'30151--dc23

2013037184

\section{British Library Cataloguing-in-Publication Data}

A catalogue record for this book is available from the British Library.

Copyright (C) 2014 by World Scientific Publishing Co. Pte. Ltd.

All rights reserved. This book, or parts thereof, may not be reproduced in any form or by any means, electronic or mechanical, including photocopying, recording or any information storage and retrieval system now known or to be invented, without written permission from the publisher.

For photocopying of material in this volume, please pay a copying fee through the Copyright Clearance Center, Inc., 222 Rosewood Drive, Danvers, MA 01923, USA. In this case permission to photocopy is not required from the publisher. 


\section{INTRODUCTION}

This volume offers an introduction to some recent developments in several active topics at the interface between geometry, topology, analysis and quantum field theory:

- Spectral geometry and index theory,

- Noncommutative models and the standard model,

- Algebraic geometry and Feynman periods,

- Generalized geometries and string theory,

- Differential geometry and gravity,

- Conformal field theory and integrability.

It is based on lectures and short communications delivered during a summer school on "Geometric, Algebraic and Topological Methods for Quantum Field Theory" held in Villa de Leyva, Colombia, in July 2011. This school was the seventh of a series of summer schools which have taken place every other year since July 1999. The invited lectures, aimed at graduate students in physics or mathematics, start with introductory material before presenting more advanced results. Each lecture is self-contained and can be read independently of the rest.

The volume begins with the lectures on Spectral Geometry by Bruno Iochum in which, strongly motivated by physics, the author presents the fundamental concepts and results in the spectral approach to noncommutative geometry. After the analytic preliminaries necessary to define the spectral action for noncommutative spectral triples, computations are carried out in some particular cases of interest in mathematical physics, such as for Riemannian manifolds (Einstein-Hilbert action) and for the noncommutative torus.

The Atiyah-Singer index theorem and some of its generalizations are the subject of the contribution by Maxim Braverman and Leonardo Cano, based on the lectures given by the first author during the summer school. The lectures start with a review of the mathematical tools required to understand the significance of the classical Atiyah-Singer index theorem, then provides a presentation of its equivariant counterpart and goes on 
to a discussion of the recent developments aimed at generalizations of the index theorem for transversally elliptic operators acting on non-compact manifolds.

The third lecture, by Paolo Aluffi, is motivated by an algebro-geometric interpretation of Feynman amplitudes in terms of periods. It starts with an informal presentation of background in algebraic geometry on the Grothendieck group of varieties and on characteristic classes, both viewed as generalizations of the ordinary topological Euler characteristic. The last part of the lecture reviews some recent work on Grothendieck classes and Chern classes of graph hypersurfaces.

The fourth lecture byJorge Zanelli is an elementary introduction to Chern-Simons forms aimed at understanding their role in physics and the reason for their usefulness in gravity. The emphasis in this presentation is on the construction of the action principles and on the geometric features that make Chern-Simons forms particularly well suited for the physics of geometric systems. The gauge invariance is reviewed, putting in the forefront the local Lorentz symmetry of space-time; the abundance of local Lorentz-invariant gravitational theories is reduced by choosing the arbitrary coefficients in the Lagrangian in such a way that, in odd dimensions, the gauge symmetry becomes enlarged.

In the fifth lecture, Matilde Marcolli covers recent applications to particle physics of models inspired by noncommutative geometry. Complementing the formal mathematical aspects of the spectral action covered by Bruno Iochum's lectures, the author discusses how an action functional for gravity coupled with matter on an ordinary space-time can be seen as pure gravity on a noncommutative product geometry. Also, it is shown how the type of noncommutative geometry involved determines the physical content of the theory, i.e. the type of fermionic and bosonic fields present in the theory. The lecture ends with an analysis of physical consequences of such particle models which include the Higgs mass prediction.

The sixth lecture by A.P. Balachandran begins with a review of the motivation for noncommutative spacetimes which arises from quantum gravity. After a physically motivated introduction to Hopf algebras, the construction of covariant quantum fields on such spacetimes is discussed. This formalism is then applied to cosmic mircowave background and to the prediction of apparent non-Pauli transitions. The lecture ends with a discussion of limits on the scale of noncommutativity, derived from experimental data.

Integrability, a way to test the so-called Maldacena conjecture, is the theme of Matthias Staudacher's Lecture. It first gives a presentation of the 
Yang Baxter equations presented as an algebraic integrability condition by means of the one dimensional spin chain and the Bethe Ansatz. The author then discusses the $A d S / C F T$ duality and its relation to integrability in a $\mathcal{N}=4$ Supersymmetric Yang-Mills Theory, after which he describes some predictions resulting from the theory.

Mariana Graña and Hagen Triendl's contribution, based on the first author's series of lectures in Villa de Leyva, provides an introduction to pure spinors, generalized complex geometry and their role in flux compactifications in the context of string theory. After a short review of some useful mathematical and physical tools in string theory and geometry, including the twisted integrability of the algebraic structure to which the pure spinor is attached, the authors give an interpretation of the manifolds arising in flux compactification in string theory from the point of view of generalized complex geometry.

The invited lectures are followed by four short communications on a wide spectrum of topics on the borderline of mathematics and physics. In their paper, Iván Contreras and Alberto Cattaneo give an overview of a novel approach to symplectic groupoids in the context of Poisson sigma models with boundary. In his contribution, Andrés Angel reviews some classical results on string topology of manifolds, and their recent extensions to the theory of orbifolds. Pedro Morales-Almazán's contribution, which discusses the Grothendieck ring class of banana and flower graphs, can be viewed as an illustration of the lectures by P. Aluffi. The last communication in this volume, by Rodrigo Vargas Le-Bert, studies the geometry underlying the non-integrable and cyclic representations of a real Lie algebra.

We hope that these self-contained and introductory but yet scientifically very challenging lectures and short communications will serve as an incentive for a broad reading audience of both mathematicians and physicists. We hope that they will motivate, as much as the school itself seems to have done, young students to pursue what might be their first acquaintance with some of the problems on the edge of mathematics and physics presented here. We further hope that the more advanced reader will find some pleasure in reading about different outlooks on related topics and seeing how the well-known mathematical tools prove to be very useful in some areas of quantum field theory.

We are indebted to various organizations for their financial support for this school. Let us first of all thank the Universidad de los Andes, which was our main source of financial support in Colombia, and the Centre International de Mathématiques Pures et Appliquées - CIMPA, for their sig- 
nificant contribution to the success of this school. Other organizations such as CLAF in Brazil, the Abdus Salam Internacional Centre for Theoretical Physics and the International Mathematical Union, through the CDE program, also contributed in a substantial way to the financial support needed for this event.

Special thanks to Mónica Castillo and Ana Catalina Salazar, who did a great job for the practical organization of the school, the quality of which was very much appreciated by participants and lecturers. We are also very indebted to Manuel Triana, Luz Malely Gutiérrez and Mauricio Morales for their help in various essential tasks needed for the successful development of the school.

We would also like to thank the administrative staff at the Universidad de los Andes, particularly Carl Langebaek, Vice-rector of Research, Carlos Montenegro, Dean of the Faculty of Sciences, Alf Onshuus, Head of the Mathematics Department, and Carlos Ávila, Head of the Physics Department, for their constant encouragement and support.

Without the people named here, all of whom helped in the organization in one way or another, before, during and after the school, this scientific event would not have left such vivid memories in the lecturers' and participants' minds. Last but not least, thanks to all the participants who gave us all, lecturers and editors, the impulse to prepare this volume through the enthusiasm they showed during the school, and thank you to all the contributors and referees for their participation in the realization of these proceedings.

The editors,

Alexander Cardona, Carolina Neira, Hernán Ocampo, Sylvie Paycha* and Andrés Reyes-Lega.

\footnotetext{
* On leave from University Blaise Pascal, Clermont-Ferrand.
} 


\section{CONTENTS}

Introduction

$\begin{array}{lll}\text { Part A LECTURES } & 1\end{array}$

Spectral Geometry $\quad 3$

B. Iochum

Index Theory for Non-compact $G$-manifolds

M. Braverman and L. Cano

Generalized Euler Characteristics, Graph Hypersurfaces, and Feynman Periods

P. Aluffi

Gravitation Theory and Chern-Simons Forms

J. Zanelli

Noncommutative Geometry Models for Particle Physics

M. Marcolli

Noncommutative Spacetimes and Quantum Physics

Integrability and the AdS/CFT Correspondence 
x Contents

\section{Part B SHORT COMMUNICATIONS}

Groupoids and Poisson Sigma Models with Boundary

A. Cattaneo and I. Contreras

A Survey on Orbifold String Topology

331

A. Angel

Grothendieck Ring Class of Banana and Flower Graphs

P. Morales-Almazán

On the Geometry Underlying a Real Lie Algebra Representation R. Vargas Le-Bert 